\title{
Aphid (Aphis craccivora Koch.) Management on Groundnut Crop (Arachis hypogaea) by using Bio-pesticides
}

\author{
Sunil Kumar Ghosh*
}

\author{
Department of Agricultural Entomology, B.C.K.V-Agricultural University, AINP on \\ Acarology, Directorate of Research, Kalyani, Nadia, West Bengal, 741235, India
}

*Corresponding author

\section{A B S T R A C T}

\section{Keywords}

Aphid distribution, Bio-pesticides, Microbial toxin, Plant extract, Organic cultivation

Article Info

Accepted:

04 September 2020 Available Online: 10 October 2020
Groundnut (Arachis hypogaea L.) family (Fabaceae) is a legume crop grown for its edible seeds. The plant is damaged by various insect pests of which Aphis craccivora Koch. causes heavy damage to tender leaves of the plant and reduces its yield. The incidence of aphid was higher on the upper and middle canopy of the groundnut plant. Imidacloprid was found most effectively against aphids providing $85.47 \%$ suppression, closely followed by mixed formulation Azadirachtin+tobacco providing 79.94\% suppression and imidacloprid + Polygonum (79.18\% suppression). From over all observation it was found that mixed formulation Azadiractin+ polygonum, microbial toxin spinosad, botanical pesticide Azadiractin, tobacco leaf extract, extracts of Polygonum floral parts gave moderate to higher results, recording about $76.09 \%, 75.07 \%, 71.77 \%, 57.59 \%$ and $55.35 \%$ aphids suppression respectively. When plant extract is mixed with plant based insecticides like azadiractin or small amount of chemical insecticide it provides better aphid control on groundnut. Plant extracts (bio-pesticides) having less or no hazardous effects on environment can be incorporated in pest management.

\section{Introduction}

Groundnut (Arachis hypogaea L.) family (Fabaceae) is grown for its edible seeds. It is an important legume crop of high nutritive value as well as being a source of edible oil. Aphis craccivora prefers to feed on legume crops; it is commonly known as "cowpea aphid." This is a serious pest on cowpea, groundnut, yard-long bean, and peanut starting from the seedling stage to podproducing stage. It acts as a direct pest and also transmits virus diseases. The pest multiplies most rapidly in late spring and with the increase in temperature, its population increases (Singh et al., 2000). Severe aphid infestations cause stunting, crinkling and curling of leaves, delayed flowering, resulting in yield reduction. Large populations of the pest secrete substantial quantities of honeydew, which favours the growth of sooty mould on leaves and reduces the photosynthetic efficiency of the plants.

Farmers become increasingly dependent on chemical pesticides to which pests have 
developed resistance (Osteen and Szmedra, 1989). A. craccivora can develop resistance to pesticides (Mokbel and Mohamed 2009). The misuse and overuse of pesticides has lead to problems of pesticide resistance, resurgence and contamination of different components of the environment Dhaliwal and Kour (2010). Plant extracts act in different ways viz. insect growth regulators (IGR), feeding deterrents, repellents and confusants (Schmutterer, 1990). Seed neem oil, either alone or in combination with bio-pesticides are useful for controlling aphid (El-Hawary and Abd ElSalam 2008). Azadirachtin is considered an excellent botanical pesticide because of its biodegradability, demonstrated low toxicity to vertebrates, environmental safety, and safety to non-target organisms (Jacobson, 1989). The botanical insecticide azadirachtin was found effective against aphid, achieving $59.23 \%$ suppression at three days after soraying (Ghosh et al., 2004). Generally, the nicotine contain of the plant (Nicotiana tabacum L.) varies from 5-10\% in the leaves (Thacker, 2002). Nicotine is effective against a wide range of pests. Use of synthetic and tobacco was more economically beneficial than using synthetics alone (Opolot et al., 2006). Ghosh (2015) reported that Polygonum hydropiper flower extract at $5 \%$ concentration and tobacco leaf extract at $10 \%$ concentration gave more than $70 \%$ and $65 \%$ aphid suppression respectively. In-vivo studies in brinjal also indicated the maximum reduction of aphids (67.3-72.3\%) within 5-14 days after application of $A$. squamosa 40 EC formulation at $1.0 \%$ dose followed by $P$. pinnata 40 EC (Purkait et al., 2019). Imidacloprid 30.5 SC @ 160ml/ha and Spinosad 45 SC @ 100ml/ha gave significant population reduction of aphid over control, providing $88.73 \%$ and $63.04 \%$ control respectively (Thakoor et al., 2019). Acetamiprid was found highly efficacious against aphid and found to suppress $85.11 \%$ aphids closely followed by neem + Spilanthes
(73.29\% control) (Ghosh, 2017). Immidacloprid, was the most effective in providing more than $80 \%$ aphid suppression on som plant followed by azadirachtin $(>70 \%$ suppression) and Polygonum extract (>60\% suppression) (Ghosh et al., 2016).

The ladybird beetles (Menochilus sexmaculatus, Brumus suturalis, Harmonia dimidiate, Brumus suturalis and Coccinella septempunctata and green lacewings (Chrysoperla carnea) are efficient predators of aphids (Muniappan et al., 2012). Synthetic insecticides, DDVP and malathion caused significantly higher killing $(>50 \%)$ whereas botanical insecticide, neem caused lower killing (less than 40\%) of the Coccinella sp (Ghosh, 2016). Chakraborty and Ghosh (2010) reported that neemactin based solution was less lethal to Coccinella septempunctata. Hence, the present research was taken to evolve a technically feasible, environmentally sound and economically viable safe pest management strategy of groundnut crops by using bio-pesticides.

\section{Materials and Methods}

\section{Study and location}

The experiment was conducted at A-B Block Farm of Bidhan Chandra Krishi Viswavidyalaya located at Kalyani, Nadia, West Bengal during the year 2018-2019. The geographical details of the site are $23^{\circ} \mathrm{N}$ latitude, $89^{\circ} \mathrm{E}$ longitude and 9.75 meter above mean sea level [MSL (Thakoor et al., 2020)]. The soil was typically gangetic alluvial soil (Entisol) with sandy clay loam texture, neutral in reaction and moderate in fertility (Priyadarsini et al., 2019). The soil type of the experimental field was sandy loam with PH range 5.75 to 6.5 (Bala et al., 2015). The climate of this zone is subtropical humid with a short winter spell during December January. 
Bio efficacy evaluation of bio-pesticides against aphid (Aphis craccivora Koch.)

\section{Cultivation practices}

The experiment based on bio-pesticides was conducted with an objective to evolve a safe pest management strategy of groundnut plant due to unique pest problem under Gangetic Alluvial Region of West Bengal, India. Groundnut plants variety AK 12-24 (Bunchy variety) was grown under recommended fertilizers and cultural practices. Spacing was taken as P-P X R-R $=15 \mathrm{~cm} \mathrm{x} 30 \mathrm{~cm}$ in $5 \mathrm{~m}$ X $5 \mathrm{~m}$ sized plots containing $530-560$ plants/plot with three replications. Two season study during post-kharif in 2018and 2019 were done. The $1^{\text {st }}$ season study during post-kharif-2018 was done as, date of showing on $02 / 08 / 2018$ and date of harvesting on $23 / 12 / 2018$ and the $2^{\text {nd }}$ season study during post- kharif-2019 was done as, date of showing on 26/7/2019 and date of harvestingon10/12/2019.

\section{Treatments details}

One microbial toxin, Saccharopolyspora sponisa (Spinosad $45 \mathrm{SC}$ ), two botanical extracts (prepared by certain indigenous procedure), Tobacco (Nicotiana tabacum) leaf, and Polygonum hydropiper floral parts, one plant based insecticide formulation, azadirachtin (Nmarin1500 ppm) were evaluated and compared with the chemical insecticide, Imidacloprid (Confidor 17.8 SL). This chemical insecticide is recommended for general use for the farmers against aphid. Some combinations of treatments i.e. mixed formulations are also used.

Treatments details are as follows.

\begin{tabular}{|l|l|}
\hline Treatments & Dose ml/L (\%) \\
\hline $\begin{array}{l}\text { Saccharopolyspora sponisa } \\
\text { (Spinosad 45 SC) (T1) }\end{array}$ & $1.0 \mathrm{ml} / 3 \mathrm{~L}$ \\
\hline Tobacco (Nicotiana tabacum) (T2) & $75.00 \mathrm{ml} / \mathrm{L}(7.5 \%)$ \\
\hline Azadirachtin (Nmarin1500 ppm) (T3) & $2.5 \mathrm{ml} / \mathrm{L}$ \\
\hline Polygonum hydropiper (T4) & $75.00 \mathrm{ml} / \mathrm{L}(7.5 \%)$ \\
\hline Imidacloprid (Confidor 17.8 SL) (T5) & $1 \mathrm{ml} / 3 \mathrm{~L}$ \\
\hline Imidacloprid + Polygonum (T6) & $0.5 \mathrm{ml} / 3 \mathrm{~L}+75.00 \mathrm{ml} / \mathrm{L}(7.5 \%)$ \\
\hline Azadiractin + tobacco (T7) & $2.5 \mathrm{ml} / \mathrm{L}+75.00 \mathrm{ml} / \mathrm{L}(7.5 \%)$ \\
\hline $\begin{array}{l}\text { Azadiractin + Polygonum } \text { (T8) } \\
\text { Untreated control (T9) }\end{array}$ & $2.5 \mathrm{ml} / \mathrm{L}+75.00 \mathrm{ml} / \mathrm{L}(7.5 \%)$ \\
\hline
\end{tabular}

Preparation of plant based extracts

The polygonum (Polygonum hydropiper) floral parts, were extracted in methanol by following the standard method developed by (Mandal et al., (2016) with a minor modification. After washing with water the plant parts were dried and powdered in grinder. $50 \mathrm{~g}$ powder of plant parts were transferred separately to the conical flask (500 $\mathrm{ml}$ capacity) and field with $250 \mathrm{ml}$ methanol. The powdered material was dipped in $250 \mathrm{ml}$ of methanol. The material was allowed to stand for 72 hours with occasional stirring at room temperature. After 72 hours the extract was filtered through Whatman 42 filter paper and residues was washed three times with methanol. Tobacco (Nicotiana tabacum) is cultivated in a large scale in the locality where experiment has been done. The tobacco 
(Nicotiana tabacum) leaves were extracted in water by following the standard method developed by Ghosh and Chakraborty, (2012) with a minor modification. After washing with water the leaves were dried and powdered in a grinder. The powdered sample $(100 \mathrm{~g})$ were transferred to a container and dipped in 1 litre water. The material was allowed to stand for 72 hours with occasional stirring at room temperature. After 72 hours the extract was filtered through Whatman 42 filter paper and added $20 \mathrm{ml}$ liquid soap.

\section{Data recording}

At 11 day intervals the sprays were done. There were three sprays in this experiment. Aphid population was recorded at 3, 6 and 10 days after spraying. Five plants were selected randomly from each plot. Aphid population per leaf from bottom, middle and top leaves was recorded. The results were presented as aphid population mortality per cent with a comparison to densities found on the controlled plot. Percent control of mite over control was calculated by following the Abbott's formula (Abbott, 1925):

Percent reduction Percent reduction in treatment - Percent reduction in control $x 100$ over control $=$

\section{0 - Percent reduction in control}

randomized block design (RBD) were followed. Data were analyzed by using INDO-STAT- software for analysis of variance. Treatment means were separated by applying CD Test (critical difference) at $5 \%$ level of significance.

\section{Phytotoxicity studies}

The observations on Phyto-toxicity symptoms (viz., leaf injury, wilting, vein clearing, necrosis, epinasty and hyponasty), if any, on the crop due to application insecticides in different treatments including untreated control were recorded at 3, 6, and 10 days after spraying using the scores shown below.

\begin{tabular}{cc}
\hline \multicolumn{2}{c}{ Scores used for effect on crop health. } \\
\hline Score & Percent crop health affected \\
\hline 0 & No adverse effect \\
1 & $1-10 \%$ \\
2 & $11-20 \%$ \\
3 & $21-30 \%$ \\
4 & $31-40 \%$ \\
5 & $41-50 \%$ \\
6 & $51-60 \%$ \\
7 & $61-70 \%$ \\
8 & $71-80 \%$ \\
9 & $81-90 \%$ \\
10 & $91-100 \%$ \\
\hline
\end{tabular}

\section{Results and Discussion}

\section{Distribution of aphid population within groundnut plants}

Aphid population (Fig. 1) was most densely populated in the upper canopy $(47.22 \%$ population) followed by middle canopy (44.45\% population) and lower canopy ( $8.33 \%$ population). This result implies that aphids were most densely congregated in the young and new leaves of groundnut plant. In his findings Ghosh, (2018) supported this type of distribution.

\section{Bio-efficacy evaluation of bio-pesticides against aphid (Aphis craccivora Koch.)}

The different treatments and their persistence at different days after spraying varied significantly in their suppression of aphid populations (Table 1 and Table 2). Among the nine treatments including one untreated control (Table 2) under present study Imidacloprid was found most effectively against aphids providing $85.47 \%$ suppression, closely followed by mixed formulation Azadirachtin + tobacco providing 79.94\% suppression and imidacloprid + Polygonum (79.18 \% suppression). From over all observation it was found that mixed formulation Azadiractin + polygonum, microbial toxin spinosad, botanical pesticide Azadiractin, tobacco leaf extract, extracts of 
Polygonum floral parts gave moderate to higher results, recording about $76.09 \%$, $75.07 \%, 71.77 \%, 57.59 \%$ and $55.35 \%$ aphids suppression respectively.

Least effectiveness against aphids was recorded from Polygonum extracts. The findings are supported by Ghosh et al., (2016) and reported that imidacloprid was the most effective in providing more than $80 \%$ aphid suppression on som plant followed by azadirachtin $\quad(>70 \%$ suppression $)$ and Polygonum extract (>60\% suppression).

Three days after treatment (Table 2), Imidacloprid was found most effectively against aphids providing $85.37 \%$ suppression, closely followed by Azadirachtin + tobacco treatment providing 84.28\% suppression and Azadiractin + Polygonum (82.59\% suppression) and Imidacloprid + Polygonum (80.58 \% suppression).

There are no significant differences among these four treatments. Six days after spraying, Imidacloprid was found to be superior insecticide $(88.61 \%$ suppression) closely followed by Azadirachtin + tobacco treatment providing $81.27 \%$ suppression and Imidacloprid + Polygonum (79.62\% suppression). Imidacloprid is significantly different from all other treatments. Nine days after spraying, also Imidacloprid was found to be most effective $(82.41 \%$ suppression) against aphids, closely followed by Imidacloprid + Polygonum $\quad \mathbf{7 7 . 3 4 \%}$ suppression) and Azadiractin + tobacco (74.27\% suppression). There is no significant different between Imidacloprid and Imidacloprid+Polygonum treatments.

In the year 2018, (Table 1) Imidacloprid was found most effectively against aphids providing $85.12 \%$ suppression, closely followed by mixed formulation Azadirachtin + tobacco providing $80.96 \%$ suppression and imidacloprid + Polygonum (78.75\% suppression). From over all observation it was revealed that microbial toxin spinosad, mixed formulation Azadiractin+ polygonum, botanical pesticide Azadiractin and tobacco leaf extract gave moderate to higher results, recording about $76.73 \%, 76.43 \%, 72.03 \%$ and $58.47 \%$ and $55.35 \%$ aphid suppression respectively.

Least effectiveness against aphids was recorded from extracts of Polygonum floral parts recording 54.96\% aphid suppression. In the year 2019, (Table 1) Imidacloprid was found most effectively against aphids providing $85.82 \%$ suppression, closely followed by mixed formulation imidacloprid + Polygonum (79.61\% suppression) and Azadirachtin + tobacco providing $78.93 \%$ suppression.

From over all observation it was revealed that mixed formulation Azadiractin + polygonum, microbial toxin spinosad, botanical pesticide Azadiractin and tobacco leaf extract gave moderate to higher results, recording about $75.75 \%, 73.41 \%, 71.50 \%$ and $56.71 \%$ aphid suppression respectively. Least effectiveness against aphids was recorded from extracts of Polygonum floral parts recording $55.75 \%$ aphid suppression.

From the overall observations it was revealed that imidacloprid was found to be most effective against aphids providing more than $85 \%$ suppression closely followed by mixed formulation Azadirachtin + tobacco and imidacloprid + Polygonum providing more than $79 \%$ suppression. Asari and Nair (1973) reported that a suspension of neem seed and a dust of silica gel were the best against aphid.

Natural product such as neem oil gave good result against another sucking pest, Bemisia tabaci (Rao, et al. 1990). 
Table.1 Efficacy of bio-pesticides against Aphid (Aphis craccivora Koch.) on groundnut (2018 and 2019)

\begin{tabular}{|c|c|c|c|c|c|c|c|c|c|c|c|}
\hline \multirow[t]{2}{*}{ Treatments } & \multirow{2}{*}{$\begin{array}{c}\text { Dose } \\
\text { ml / Litre } \\
(\%)\end{array}$} & \multicolumn{5}{|c|}{ Overall efficacy ( \% reduction) 2018} & \multicolumn{5}{|c|}{ Overall efficacy ( \% reduction) 2019} \\
\hline & & $\begin{array}{c}\text { Pre- } \\
\text { Treatment } \\
\text { Obs. } \\
\text { Aphid/3Leaf }\end{array}$ & 3 DAT & 6 DAT & 10 DAT & Mean & $\begin{array}{c}\text { Pre- } \\
\text { Treatment } \\
\text { Obs. } \\
\text { Aphid/3Leaf }\end{array}$ & 3 DAT & 6 DAT & $\begin{array}{c}10 \\
\text { DAT }\end{array}$ & Mean \\
\hline $\begin{array}{c}\text { S.spinosa (Spinosad } 45 \text { SC) } \\
\text { (T1) }\end{array}$ & $1 \mathrm{ml} / 3 \mathrm{~L}$ & 10.33 & $\begin{array}{c}78.06 \\
(62.21)\end{array}$ & $\begin{array}{c}78.60 \\
(62.06)\end{array}$ & $\begin{array}{c}73.52 \\
(59.04)\end{array}$ & $\begin{array}{c}76.73 \\
(61.10)\end{array}$ & 8.67 & $\begin{array}{c}81.25 \\
(67.99)\end{array}$ & $\begin{array}{c}75.29 \\
(60.15)\end{array}$ & $\begin{array}{c}63.68 \\
(53.02)\end{array}$ & $\begin{array}{c}73.41 \\
(60.39)\end{array}$ \\
\hline $\begin{array}{c}\text { Tobacco } \\
\text { (T2) }\end{array}$ & $\begin{array}{c}75.00 \mathrm{ml} / \mathrm{L} \\
(7.5 \%)\end{array}$ & 11.07 & $\begin{array}{c}67.72 \\
(53.01)\end{array}$ & $\begin{array}{c}58.72 \\
(50.07)\end{array}$ & $\begin{array}{c}48.98 \\
(44.41)\end{array}$ & $\begin{array}{c}58.47 \\
(49.16)\end{array}$ & 8.58 & $\begin{array}{c}65.86 \\
(54.24)\end{array}$ & $\begin{array}{c}56.83 \\
(48.96)\end{array}$ & $\begin{array}{c}47.43 \\
(43.47)\end{array}$ & $\begin{array}{c}56.71 \\
(48.89)\end{array}$ \\
\hline $\begin{array}{c}\text { Azadirachtin } \\
\text { (Nimarin } 1500 \text { ppm) (T3) }\end{array}$ & $2.5 \mathrm{ml} / \mathrm{L}$ & 10.96 & $\begin{array}{c}78.68 \\
(62.58)\end{array}$ & $\begin{array}{c}75.10 \\
(60.13)\end{array}$ & $\begin{array}{c}62.31 \\
(52.42)\end{array}$ & $\begin{array}{c}72.03 \\
(58.38)\end{array}$ & 9.12 & $\begin{array}{c}75.09 \\
(60.04)\end{array}$ & $\begin{array}{c}73.17 \\
(58.36)\end{array}$ & $\begin{array}{c}66.27 \\
(54.27)\end{array}$ & $\begin{array}{c}71.51 \\
(57.56)\end{array}$ \\
\hline Polygonum (T4) & $\begin{array}{c}75.00 \mathrm{ml} / \mathrm{L} \\
(7.5 \%)\end{array}$ & 10.98 & $\begin{array}{c}63.64 \\
(52.95)\end{array}$ & $\begin{array}{c}53.70 \\
(47.13)\end{array}$ & $\begin{array}{c}47.53 \\
(43.37)\end{array}$ & $\begin{array}{c}54.96 \\
(47.82)\end{array}$ & 8.67 & $\begin{array}{c}62.93 \\
(52.53)\end{array}$ & $\begin{array}{c}56.83 \\
(48.96)\end{array}$ & $\begin{array}{c}47.50 \\
(42.71)\end{array}$ & $\begin{array}{c}55.75 \\
(48.07)\end{array}$ \\
\hline $\begin{array}{c}\text { Imidaclorprid } \\
\text { (Confidor 17.8 SL) (T5) }\end{array}$ & $1 \mathrm{ml} / 3 \mathrm{~L}$ & 11.01 & $\begin{array}{c}83.27 \\
(65.62)\end{array}$ & $\begin{array}{c}87.75 \\
(69.72)\end{array}$ & $\begin{array}{c}84.33 \\
(67.15)\end{array}$ & $\begin{array}{c}85.12 \\
(67.50)\end{array}$ & 9.92 & $\begin{array}{c}87.47 \\
(68.97)\end{array}$ & $\begin{array}{c}89.48 \\
(71.06)\end{array}$ & $\begin{array}{c}80.50 \\
(63.42)\end{array}$ & $\begin{array}{c}85.82 \\
(67.82)\end{array}$ \\
\hline $\begin{array}{c}\text { Imidacloprid+Polygonum } \\
\text { (T6) }\end{array}$ & $\begin{array}{l}0.5 \mathrm{ml} / 3 \mathrm{~L}+ \\
75.00 \mathrm{ml} / \mathrm{L}\end{array}$ & 11.30 & $\begin{array}{c}79.77 \\
(62.67)\end{array}$ & $\begin{array}{c}80.40 \\
(65.01)\end{array}$ & $\begin{array}{c}76.09 \\
(60.42)\end{array}$ & $\begin{array}{c}78.75 \\
(62.70)\end{array}$ & 9.63 & $\begin{array}{c}81.40 \\
(66.01)\end{array}$ & $\begin{array}{c}78.84 \\
(61.56)\end{array}$ & $\begin{array}{c}78.60 \\
(62.07)\end{array}$ & $\begin{array}{c}79.61 \\
(63.21)\end{array}$ \\
\hline $\begin{array}{c}\text { Azadiractin+tobacco } \\
\text { (T7) }\end{array}$ & $\begin{array}{l}2.5 \mathrm{ml} / \mathrm{L}+ \\
75.00 \mathrm{ml} / \mathrm{L}\end{array}$ & 11.17 & $\begin{array}{c}85.33 \\
(67.61)\end{array}$ & $\begin{array}{c}82.09 \\
(65.44)\end{array}$ & $\begin{array}{c}75.46 \\
(60.45)\end{array}$ & $\begin{array}{l}80.96 \\
(64.5)\end{array}$ & 8.87 & $\begin{array}{c}83.24 \\
(66.36)\end{array}$ & $\begin{array}{c}80.45 \\
(65.03)\end{array}$ & $\begin{array}{c}73.09 \\
(58.42)\end{array}$ & $\begin{array}{r}78.93 \\
(63.27)\end{array}$ \\
\hline $\begin{array}{c}\text { Azadiractin+Polygonum } \\
\text { (T8) }\end{array}$ & $\begin{array}{l}2.5 \mathrm{ml} / \mathrm{L}+ \\
75.00 \mathrm{ml} / \mathrm{L}\end{array}$ & 10.27 & $\begin{array}{c}81.27 \\
(67.98)\end{array}$ & $\begin{array}{c}78.84 \\
(61.56)\end{array}$ & $\begin{array}{c}69.17 \\
(56.23)\end{array}$ & $\begin{array}{c}76.43 \\
(61.92)\end{array}$ & 9.67 & $\begin{array}{c}83.92 \\
(66.49)\end{array}$ & $\begin{array}{c}75.39 \\
(60.56)\end{array}$ & $\begin{array}{c}67.93 \\
(55.54)\end{array}$ & $\begin{array}{c}75.75 \\
(60.86)\end{array}$ \\
\hline Untreated Control (T9) & --- & 10.67 & $\begin{array}{c}0.00 \\
(4.05)\end{array}$ & $\begin{array}{c}0.00 \\
(4.05)\end{array}$ & $\begin{array}{c}0.00 \\
(4.05)\end{array}$ & --- & 8.75 & $\begin{array}{c}0.00 \\
(4.05)\end{array}$ & $\begin{array}{c}0.00 \\
(4.05)\end{array}$ & $\begin{array}{c}0.00 \\
(4.05)\end{array}$ & --- \\
\hline $\begin{array}{l}\text { S Em }( \pm) \\
\text { CD at } 5 \%\end{array}$ & --- & NS & $\begin{array}{l}1.60 \\
4.95\end{array}$ & $\begin{array}{l}1.78 \\
5.33\end{array}$ & $\begin{array}{l}1.76 \\
5.28\end{array}$ & $\begin{array}{l}-- \\
---\end{array}$ & NS & $\begin{array}{l}1.50 \\
4.64\end{array}$ & $\begin{array}{l}1.54 \\
4.88\end{array}$ & $\begin{array}{l}1.69 \\
5.03\end{array}$ & $\begin{array}{l}-- \\
---\end{array}$ \\
\hline
\end{tabular}

Figure in the parenthesis are angular transformed values, DAT $=$ Days after treatment, NS = Not significant 
Table.2 Overall efficacy of bio-pesticides against Aphid (Aphis craccivora Koch.) on groundnut (Grand Mean of 2018 and 2019)

\begin{tabular}{|c|c|c|c|c|c|c|}
\hline \multirow[t]{2}{*}{ Treatments } & \multirow{2}{*}{$\begin{array}{c}\text { Dose } \\
\text { ml / Litre } \\
(\%)\end{array}$} & \multicolumn{5}{|c|}{ Overall efficacy (Grand Mean of 2010 and 2011) } \\
\hline & & $\begin{array}{l}\text { Pre-Treatment Obs. } \\
\text { Aphid/3Leaf }\end{array}$ & 3 DAT & 6 DAT & 10 DAT & Mean \\
\hline $\begin{array}{c}\text { S.spinosa (Spinosad 45 SC) } \\
\text { (T1) }\end{array}$ & $1 \mathrm{ml} / 3 \mathrm{~L}$ & 9.50 & $\begin{array}{l}79.65 \\
(65.1)\end{array}$ & $\begin{array}{c}76.94 \\
(61.10)\end{array}$ & $\begin{array}{c}68.60 \\
(56.03)\end{array}$ & $\begin{array}{c}75.07 \\
(60.74)\end{array}$ \\
\hline $\begin{array}{c}\text { Tobacco } \\
\text { (T2) }\end{array}$ & $75.00 \mathrm{ml} / \mathrm{L}(7.5 \%)$ & 9.82 & $\begin{array}{c}66.79 \\
(53.62)\end{array}$ & $\begin{array}{c}57.77 \\
(49.51)\end{array}$ & $\begin{array}{c}48.20 \\
(48.20)\end{array}$ & $\begin{array}{c}57.59 \\
(49.02)\end{array}$ \\
\hline $\begin{array}{c}\text { Azadirachtin } \\
\text { (Nimarin } 1500 \text { ppm) (T3) }\end{array}$ & $2.5 \mathrm{ml} / \mathrm{L}$ & 10.04 & $\begin{array}{c}76.88 \\
(61.31)\end{array}$ & $\begin{array}{c}74.13 \\
(59.24)\end{array}$ & $\begin{array}{c}64.29 \\
(53.34)\end{array}$ & $\begin{array}{c}71.77 \\
(57.97)\end{array}$ \\
\hline Polygonum (T4) & $75.00 \mathrm{ml} / \mathrm{L}(7.5 \%)$ & 9.82 & $\begin{array}{c}63.28 \\
(52.74)\end{array}$ & $\begin{array}{c}55.26 \\
(48.04)\end{array}$ & $\begin{array}{c}47.51 \\
(43.04)\end{array}$ & $\begin{array}{c}55.35 \\
(47.94)\end{array}$ \\
\hline $\begin{array}{c}\text { Immidachlorprid } \\
\text { (Confidor 17.8 SL) (T5) }\end{array}$ & $1 \mathrm{ml} / 3 \mathrm{~L}$ & 10.46 & $\begin{array}{r}85.37 \\
(67.27)\end{array}$ & $\begin{array}{c}88.61 \\
(70.39)\end{array}$ & $\begin{array}{c}82.41 \\
(65.28)\end{array}$ & $\begin{array}{c}85.47 \\
(67.66)\end{array}$ \\
\hline $\begin{array}{c}\text { Imidacloprid+Polygonum } \\
\text { (T6) }\end{array}$ & $0.5 \mathrm{ml} / 3 \mathrm{~L}+75.00 \mathrm{ml} / \mathrm{L}$ & 10.46 & $\begin{array}{c}80.58 \\
(64.34)\end{array}$ & $\begin{array}{c}79.62 \\
(63.28)\end{array}$ & $\begin{array}{c}77.34 \\
(61.24)\end{array}$ & $\begin{array}{r}79.18 \\
(62.95)\end{array}$ \\
\hline $\begin{array}{c}\text { Azadiractin+tobacco } \\
\text { (T7) }\end{array}$ & $2.5 \mathrm{ml} / \mathrm{L}+75.00 \mathrm{ml} / \mathrm{L}$ & 10.02 & $\begin{array}{r}84.28 \\
(66.98)\end{array}$ & $\begin{array}{c}81.27 \\
(65.23)\end{array}$ & $\begin{array}{c}74.27 \\
(59.43)\end{array}$ & $\begin{array}{r}79.94 \\
(63.88)\end{array}$ \\
\hline $\begin{array}{c}\text { Azadiractin+Polygonum } \\
\text { (T8) }\end{array}$ & $2.5 \mathrm{ml} / \mathrm{L}+75.00 \mathrm{ml} / \mathrm{L}$ & 9.97 & $\begin{array}{c}82.59 \\
(67.23)\end{array}$ & $\begin{array}{c}77.11 \\
(61.06)\end{array}$ & $\begin{array}{c}68.55 \\
(55.88)\end{array}$ & $\begin{array}{r}76.09 \\
(61.39)\end{array}$ \\
\hline Untreated Control (T9) & --- & 9.71 & $\begin{array}{c}0.00 \\
(4.05)\end{array}$ & $\begin{array}{c}0.00 \\
(4.05)\end{array}$ & $\begin{array}{c}0.00 \\
(4.05)\end{array}$ & --- \\
\hline $\begin{array}{l}\text { S Em }( \pm) \\
\text { CD at } 5 \%\end{array}$ & $\begin{array}{l}--- \\
---\end{array}$ & NS & $\begin{array}{l}1.55 \\
5.37\end{array}$ & $\begin{array}{l}1.74 \\
5.10\end{array}$ & $\begin{array}{l}1.77 \\
5.20\end{array}$ & $\begin{array}{l}--- \\
---\end{array}$ \\
\hline
\end{tabular}

Figure in the parenthesis are angular transformed values, DAT = Days after treatment, NS = Not significant 
Fig.1

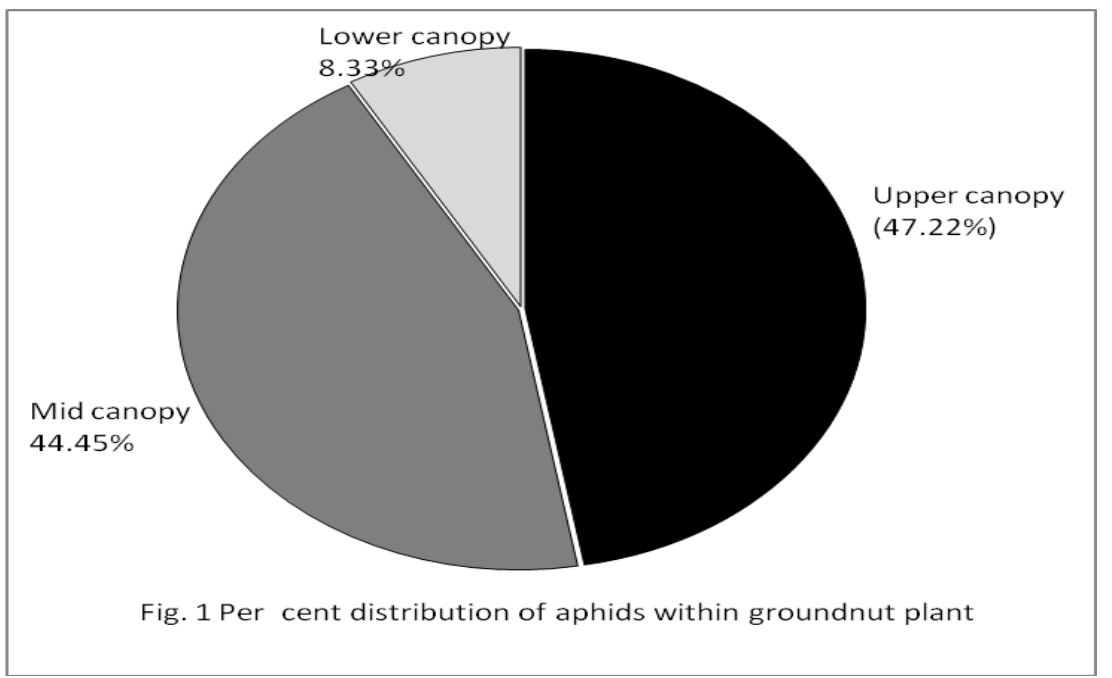

These findings support the activity of neem products. However, extracts of Polygonum plant gave better aphids control, recording more than $60 \%$ suppression which is supported by Ghosh et al., (2009) where they reported that polygonum plant extracts provided $59.77 \%$ aphid suppression in ladysfinger field. But Imidacloprid is a highly toxic synthetic chemical insecticide, so there is every possibility to contaminate food materials with the toxic chemicals. Mixed formulation of bio-pesticides like Azadirachtin + tobacco and Azadiractin + polygonum or mixed formulation of small amount imidacloprid with polygonum provided higher aphid control at par with the inidacloprid in its recommended doses. So we can avoid the use of highly toxic insecticides like imidacloprid and other chemical insecticides. Das et al., (2010) and Ghosh et al., (2012 and 2013) reported that a rapid degradation of persistency was observed in imidacloprid and neem oil than other pesticides tested. So imidacloprid as small amount may be recommended mixing with plant based insecticides for general use of the farmers for its higher efficacy and rapid degradation. We can use plant based insecticides with plant extract as mixed formulations. Plant based insecticides or plant extract cannot give higher control when it is used individually but when it is mixed with other formulations it provides higher control. We also can use plant based insecticides mixing with very small dose of chemical insecticides like imidacloprid with azadiractin/polygonam/tobacco extracts which will be eco-friendly.

\section{Phytotoxicity studies}

No phytotoxic symptoms were observed as irrespective doses of different treatments and untreated control in respect of leaf injury, wilting, vein clearing, necrosis, epinasty and hyponasty. So bio-pesticides under the study are highly recommended.

Aphid populations are mostly congregated on upper and middle canopy recording $47.22 \%$ and $44.45 \%$ respectively. So for aphid control special care should be taken to spray on upper and middle canopy of groundnut crop. Mixed formulation Azadirachtin + tobacco providing $79.94 \%$ suppression and imidacloprid + Polygonum $(79.18 \%$ suppression) which are at par with the chemical insecticide, imidacloprid. There is no significant difference among these 
treatments. Small amount of chemical insecticide mixing with bio-pesticides are also less harmful. So they also are included for aphid control. These treatment have no phytotoxic effect. Bio-pesticides used under the present study are of biological origin having low or no hazardous effect on environment and health so can be incorporated in IPM programme for aphid management.

\section{Acknowledgements}

This study was carried out with the support of the Director of research and Department of Agricultural Entomology, BCKV, and the Coordinator, AINP on Acarology for providing supports and facilities. I thank the Department, as well as all those who have contributed to it.

\section{References}

Abbott, W. S. (1925). A method of computing the effectiveness of an insecticide. $J$. Econ. Entomol., 18, 265-267.

Asari, P. A. R. and Nair, M. R. G. K. (1973). On the control of brinjal pests using deterrents. Agric. Res. J. Kerala, 10(2): $133-135$.

Bala, S. C., Karmakar, K.. and Ghosh, S.K. (2015). Population dynamics of mite, Aceria tulipae Keif. On garlic (Allium sativum L.) and its management under Bengal basin. International Journal of Science, Environment and Technology. 4 (5): 1365-1372.

Chakraborty, K. and Ghosh, S.K. (2010). Incidence of Coccinella septempunctata in brinjal with some pesticides. Current advances in Agricultural Sciences, 2(2): 129-130.

Das, K., Biswas, S., Chakraborty,G. and Ghosh, S.K. (2010). Efficacy of insecticides against Iassid (Amrasca biguttula biguttuka Ishida) on okra in terai agro-ecology of West Bengal. $J$. Appl. Zool. Res., 21 (1): 33-35.

Dhaliwal, G. S. and Koul, O. (2010). Quest for Pest Management: From Green Revolution to Gene Revolution. Kalyani Publishers, New Delhi.

El-Hawary F. M., Abd El-Salam A.M.E. (2008). Effect of neem and antitranspirant products against Aphis craccivora Koch and its biology. Egypt. Acad. J. Biolog. Sci. 1(2):189-196.

Ghosh, S. K. (2015). Integrated field management of aphid (Myzus persicae Sulz. And Aphis gossypii Glov. Together) on potato (Solanum tuberosum L.) using bio-pesticides International Journal of Science, Environment and Technology. 4 (3): 682-689.

Ghosh, S. K. (2016). Harmful effect of insecticides against predator, Coccinella sp. (Lady bird beetle) on eggplant (Solanum melongena L.). Uttar Pradesh J. Zool. 36(1): 17-23.

Ghosh, S. K. (2017). Seasonal Incidence of aphid (Aphis gossypii Glove.) Infesting tomato (Lycopersicon esculentum L.) and their management by using botanical pesticides International Journal of Advances in Science Engineering and Technology. 5(3, Spl. Issue-1):14-17.

Ghosh, S. K. (2018). Phytochemicals-A New Era for Management of Red Spider Mite (Tetranychus urticae Koch.) on Rose Plant (Rosa chinensis L.) Book Edited by Dr. S.S. Gantait, Book title "Advances in Floriculture and Urban Horticulture" pp. 300-304, 2018.

Ghosh, S. K. and Chakraborty, G. (2012). Integrated field management of Henosepilachna vigintioctopunctata (Fabr.) on potato using botanical and microbial pesticides. Journal of biopesticides, 5 (supplementary): 151154. 
Ghosh, S. K., LaskaI, N. and Senapati, S. K. (2004). Seasonal fluctuation of Aphis gossypii Glov. on brinjal, md field evaluation of pesticides from different origin against $A$. gossypii under tcrai region. Indian J Agric. Res. 38(3):171177.

Ghosh, S. K., Mahapatra, G.S.S. and Chakraborty, G. (2009). Field efficacy of plant extracts and microbial insecticides against aphid (Aphis gossypii) infesting okra (Abelmoschus esculentus). Redia, Itali XC11: 249252. (with sub-title Journal of Entomology).

Ghosh, S. K., Mandol, T. and Chakraborty, K. (2016). Population fluctuation of aphid (Aphis craccivora Koch.) infesting Som plant leaves (Machilus bombycina King..) and its management. $J$. Ent.Res.. 40 (3): 235-241.

Ghosh, S. K., Mandal, T., Biswas, S. and Chakraborty, K. (2012). Field evaluation of cultivars and bio-efficacy of insecticides against pest complex of ladysfinger (Abelmoschus esculentus L.). Journal of applied Zoological research 23(2): 121-128

Ghosh, S. K., Mandal, T. and Chakraborty, K. (2013). Efficacy of chemical insecticides and neem oil against white fly (Bemisia tabaci Genn.) Infesting ladysfinger (Abelmoschus esculentus L.). International Journal of Bioresource and Stress Management, 4 (2): special 348-351.

Jacobson, M. (1989). Pharmacology and Toxicology of neem. In: Phytochemical Pesticides, Vol. 1. The Neem Tree, M. Jacobson (eds.) CRC Press, Boca Raton, FL, pp. 133-153.

Mandol, T., Ghosh, S. K. and Chakraborty, K. (2016) Seasonal incidence of thrips (Thrips tabaci L.) infesting Som plant leaves (Machilus bombycina King..) and their management using bio- pesticides International Journal of Science, Environment and Technology. 5 (4): 2245-2256.

Mokbel, El-Sayed S. and Azza I. Mohamed (2009). Development of resistance in field strain of Aphis craccivora to the donotefuran insecticides from the new class neonicotinoids and its effects on some enzymes control. Egypt Acad. J. Biolog. Sci., 1 (1): 65-69.

Muniappan, R., Shepard, B. M., Carner, G. R. and Ooi, P.A.C. (2012). Arthropod pests of horticultural crops in tropical Asia. Wallingford, Oxfordshire, CABI.

Opolot, H. N., Agona, A., Kyamanywa, S., Mbata, G. N. and Adipala, E. (2006). Integrated field management of cowpea pests using selected synthetics and botanical pesticides. Crop Prot., 25 (11): 1145-1152.

Osteen, C. D. and Szmedra, P. I. (1989). Agricultural Pesticide Use Trends and Policy Issues. Agricultural Economic Report No. 622, Department of Agriculture, Washington, D.C., U.S.

Priyadarshini, S., Ghosh, S. K and Nayak, A.K. (2019). Field screening of different chilli cultivars against important sucking pests of chilli in West Bengal. Bulletin of Environment, Pharmacology and Life Sciences. (JEZS) 8(7): 134-140.

Purkait, A., Biswas, S., Saha, S., Hazra, D. K., Roy, K., Biswas, P. K., Ghosh, S. K. and Kole, R. K. (2019).Formulation of plant based insecticides, their bioefficacy evaluation and chemical characterization. Crop Protection, 125, 104907, 1-9.

Rao, N. V., Reddy, A. S. and Reddy, P. S. (1990). Relative efficacy of some new insecticides on insect pests of cotton. Indian J. Plant Prot. 18(1): 53 - 58.

Schmutterer, H. (1990). Properties and potential of natural pesticides from the neem tree, Azadirachta indica. Ann. 
Rev. Entomol., 35: 271-297.

Singh, R. N., Samson, M. V. and Dutta, R. K. (2000). Pest management in sericulture. Indian Publishers Distribution, pp. 50270.

Thacker, J.R.M. (2002). An Introduction to Arthropod Pest Control. Cambridge University Press, Cambridge.

Thakoor, P., Ghosh, S. K., Nihal, R. and Ramya Sri, N. (2019). Effect of abiotic factors on seasonal incidence and bioefficacy of some newer insecticides against aphid (Aphis gossypii) in tomato. Journal of Entomology and Zoology studies. 7(3): 513-516.

Thakoor, P., Ghosh, S.K. and Bala, S.C. (2020) Effect of abiotic factors on seasonal incidence and bio-efficacy of some newer insecticides against white fly on tomato crop in West Bengal. Journal of Entomology and Zoology studies. 8(3): 267-271.

\section{How to cite this article:}

Sunil Kumar Ghosh. 2020. Aphid (Aphis craccivora Koch.) Management on Groundnut Crop (Arachis hypogaea) by using Bio-pesticides. Int.J.Curr.Microbiol.App.Sci. 9(10): 24-34.

doi: https://doi.org/10.20546/ijcmas.2020.910.004 